International Journal on Cybernetics \& Informatics (IJCI) Vol. 5, No. 2, April 2016

\title{
DIGITAL RECEIVER SUBSYSTEM USING DDSFREQUENCY SYNTHESIZER
}

\author{
Ahalya R S ${ }^{1} \&$ Mary Joseph ${ }^{2}$ \\ ${ }^{1}$ Department of electronics, Mar Athanasius College Of Engineering, \\ A P J Abdul Kalam Technological University, Kerala, India \\ ${ }^{2}$ Associate Professor, Department of Electronics \&Communication Engineering \\ M.A.College of Engineering, Kothamangalam
}

\begin{abstract}
An important aspect of Electronic Warfare is the development of Radar Warning Receivers (RWR). Radar Warning Receiver/Electronic Warfare Receiver provides superior performance to protect tactical, transport and special mission aircraft in today's modern electronic combat environment. Radar warning receiver ( $R W R$ ) systems detect the radio emissions of radar systems. Their purpose is to issue a warning when a radar detects a threat. The warning can then be used, to find out the detected threat.The performance and design aspects of radar receivers will be considered in this project. For this purpose, the radar receiver will be defined as that assemblage of components within the radar system which is required to detect, amplify, and present the desired information as gathered at the radar location. This project is focused mainly on electronic warfare radar systems and describes the receiver front end design, as well as the software algorithms used to determine signal characteristics.
\end{abstract}

\section{KEYWORDS}

RWR, RF signals, pulsed radars,DFT,FFT

\section{INTRODUCTION}

In Electronic Warfare (EW), radar is used to detect vehicles, ships, and aircraft, and to guide weaponry. Radars can also be used to detect threats and can be called as Electronic Counter Measures. For every newly developed threat, there is the need to neutralize that threat. This is a computer controlled radar warning receiver (RWR) that provides detection and display of RF signals from the threats. The digital system usually includes time-frequency transfer. The following parameters are used to identify and locate the origin of the threat signal:

DOI: $10.5121 /$ ijci.2016.5233 
International Journal on Cybernetics \& Informatics (IJCI) Vol. 5, No. 2, April 2016

\section{- $\quad$ FREQUENCY}

\section{- $\quad$ PULSE WIDTH}

\section{- $\quad$ PULSE REPETITION RATE}

For the scope of this project, it determines the initial detection and classification of the radar signal. The frequency, pulse width, and pulse repetition rate are the parameters, which are to befind out. A radio frequency (RF) signal is given to the Digital Radar Receiver and processed, in order to determine the above three parameters. Future applications to this project are to further analyze and manipulate the received RF signal to jam the enemy's communication systems. Here it can be as simple as detecting the presence of energy in a specific radar band[2]. For more critical condiions, such as military, the systems are often capable of classifying the source of the radar by the signal's strength[5], phase and waveform type, like pulsed power wave or continuous wave with amplitude modulation or frequency modulation[4]. The signal's strength and waveform will be helpful to estimate the most probable type of threat the detected radar possess.

\section{WORKING PRINCIPLE}

The Radar warning receivers functional elements can be divided into six elements.

Reception : interfacing the equipment to electromagnetic environment.

Conversion : amplification, up/down converting and filtering.

Detection : receiver specific signal detection and measurement.

Extraction : extraction of signal characteristics from time and frequency domain.

Analysis/control :deinterleaving signal characterization and identification.

Indication : display generation and parameter distribution.

The RF signal from the target is initially down converted to IF signal and the analogue input signal is transferred into Time discrete amplitude samples. Maximum IF bandwidth is defined by sampling frequency and it must be greater than 2 times IF bandwidth. Then the Time domain samples are combined into frames each of $\mathrm{N}$ samples. These samples are used to perform a Fast Fourier Transformation (FFT). Time effective calculation can be performed if number of samples $=2^{\mathrm{N}}$. And then the analog signal is converted to pulse descriptor words (PDW). Then for each valid signal, the information such as pulse width, coarse and fine frequency, signal amplitude, phase etc are derived from pulse descriptor words. These parameters are derived using parallel and sequential correlation. The product of sampling rate and FFT length defines the Time 
Resolution. Frequency selection and $\mathrm{S} / \mathrm{N}$ can be improved and time resolution can be decreased with higher FFTs.

\section{SYSTEM DESCRIPTION}

\subsection{Block Diagram}

The system block diagram is shown in figure. The user input is a software input, which is used to set the LO range. The pulse radar RF signal is mixed with the frequency synthesizer signal, which acts like a local oscillator. The internal signal, IF, is the down converted pulse radar signal which is fed into the A/D board which is connected to the PC. Then the signal is processed using DSP methods, and if a radar signal is present the signals characteristics are sent as outputs.

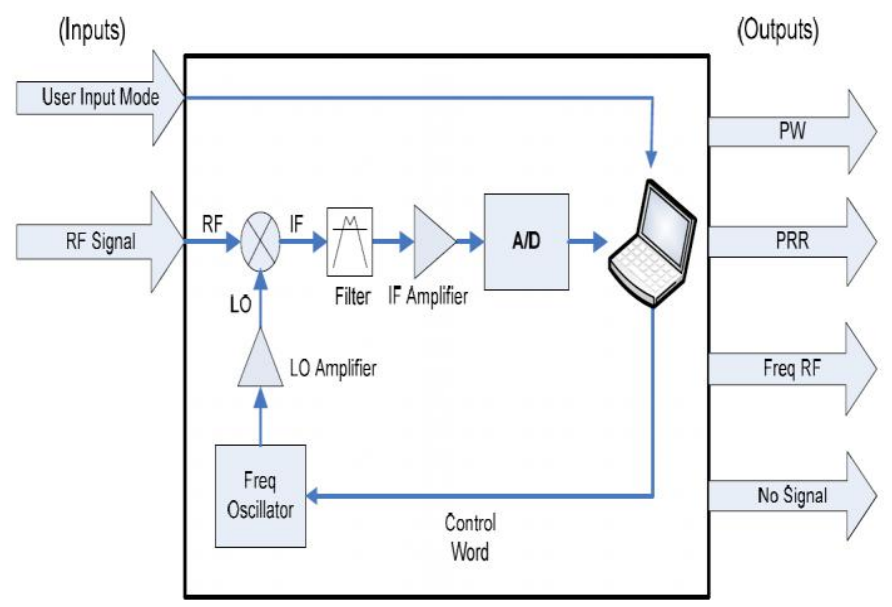

Fig:1 Block Diagram

The function of the system is to detect a pulsed radar signal over a large frequency and power range. The frequency ranges from 50 to $120 \mathrm{MHz}$ and the power level can be as low as $-20 \mathrm{dBm}$. The mixer multiplies the incoming RF signal of range $50-120 \mathrm{MHz}$ with the LO signal and produces the down converted IF signal. A control word is sent to frequency synthesizer through the parallel port, which determines its output. In order to boost the signal from the frequency synthesizer a local amplifier is used. The amplifier is needed to get the amplitude from the frequency synthesizer up to the $7 \mathrm{dBm}$ level needed for the mixer. This signal is fed to the mixer. Thus the signal output from the mixer is adapted to detector IF range. Then the down converted signal is conditioned and for that the signal is then fed to a low pass filter of cut off frequency 200 $\mathrm{kHz}$. after eliminating noise the filtered signal is fed to an amplifier which make up the signal loss 
encountered in various parts of the system and also compensate the losses due to filter. The amplifier is used to set the voltage level of IF signal for the best data acquisition. And then conditioned signal is then fed to analog to digital converter. The ADC converts the analog signal to Pulse Descriptor Words which the information about pulse width, amplitude, phase and frequency of the input radar signal. The PDW from ADC (Data acquisition card) is fed to processor and the signal is processed using DSP methods. The signal processing involves taking the FFT of the signal and detecting whether a radar signal is present or not. If the radar signal is absent it outputs no signal and the frequency synthesizer output is incremented and if a radar signal is present it outputs the information about pulse width, pulse repetition rate and frequency of the input radar signal.

Data acquisition subsystem consists of an analog filter, amplifier, and the data acquisition board. The IF signal is filtered using a low-pass filter with a cut off frequency $200 \mathrm{Khz}$, which passes the intermediate frequencies with little attenuation, but attenuates signals with frequencies above 200 KHz. After filtering, the signal is amplified using a 741 amplifier. The amplifier can add a $20 \mathrm{~dB}$ of gain to compensate for the attenuation caused by the filter.

\subsubsection{Frequency Synthesizer}

A frequency synthesizer is a system that generates a number of precise frequencies from a single reference frequency.

A frequency synthesizer can replace the array of crystal resonators in a multichannel radio receiver frequency synthesizers are relatively inexpensive and can be easily controlled by digital circuitry. Hence they are used in new communication system designs. Frequency synthesizers are found in many devices, including radio receivers, mobile telephones, radiotelephones, walkietalkies, satellite receivers, GPS systems, etc. to obtain the required output signal it can perform frequency multiplication and frequency division. The control word is sent to the frequency synthesizer, through the parallel port, and it decides the frequency of the synthesizer output. Foe power boosting a10 $\mathrm{dB}$ amplifier is used. The amplifier should get a amplitude up to $7 \mathrm{dBm}$ from the DDS frequency synthesizer for the mixer. The DDS 9854 is used as the frequency synthesizer. It uses a technique called direct digital synthesis creating a stable sinusoidal wave form. The maximum frequency of the synthesizer is $120 \mathrm{MHz}$ to prevent aliasing. 
International Journal on Cybernetics \& Informatics (IJCI) Vol. 5, No. 2, April 2016

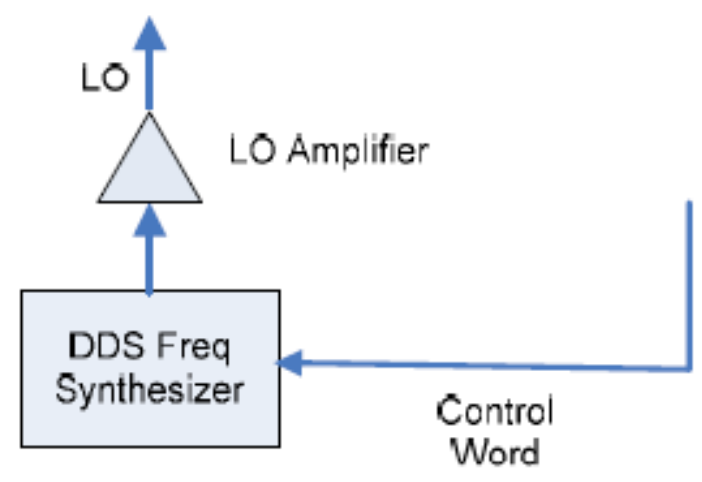

Fig 2 Frequency synthesizer subsystem

\subsection{DSP Flow chart}

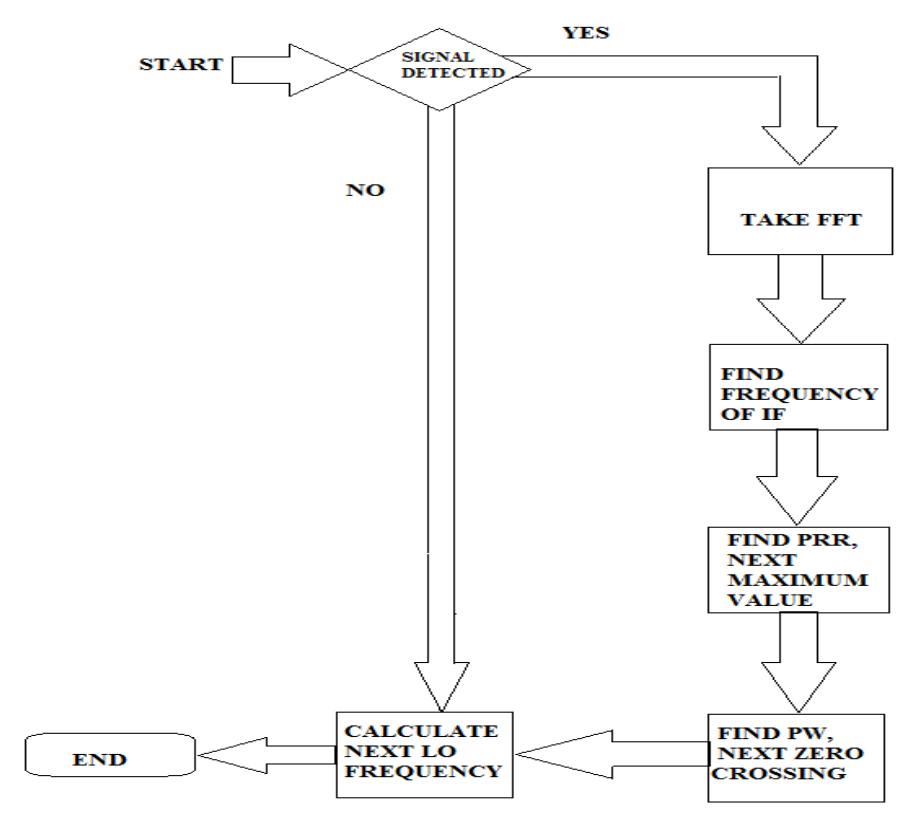

Fig: 3 DSP FLOW CHART

A fast Fourier transform (FFT) is an algorithm to find out the discrete Fourier transform (DFT) and its inverse. A fast Fourier transform converts time to frequency and vice versa; an FFT rapidly computes such transformations by factorizing the DFT matrix into a product of mostly zero factors. 
International Journal on Cybernetics \& Informatics (IJCI) Vol. 5, No. 2, April 2016

The DFT is obtained by decomposing a sequence of values into components of different frequencies. An FFT is a way to compute the DFT of $N$ points. FFTs are of great importance to a wide variety of applications, from digital signal processing and solving partial differential equations to algorithms for quick multiplication of large integers.

Figure 3 shows how the radar signal is processed in Mat lab.

\footnotetext{
$>\quad$ The process checks whether a signal is detected

$>\quad$ If there is no signal, the frequency synthesizer is incremented and the program exits.

$>\quad$ If a signal is there, then its FFT is calculated.

$>\quad$ The FFT will be having an array whose points correspond to various magnitudes.

$>\quad$ A frequency array is made based on the number of points in the FFT and the sampling rate.

$>\quad$ To calculate the IF frequency the point with the maximum value in FFT array is found and is correlated with same point.

$>\quad$ To calculate the PRR the point with largest magnitude next to previous value is found.

$>\quad$ PRR is obtained by subtracting this value from he IF frequency.

$>\quad$ Value of local minimum when subtracted from the IF frequency gives the pulse width.
}

\section{RESULTS AND DISCUSSIONS}

Before signal processing signal has two level of detection. Figure illustrates this detection process. The first level1 detection checks whether the signal's amplitude is above the preset threshold value. The threshold value is $200 \mathrm{mV}$. It corresponds to about $-20 \mathrm{Db} \mathrm{m}$ signal level on the input. Once Level 1 is over, Level 2 Detection starts. During this level Detection the system takes an FFT with a small number of points and then searches for the spikes on either side of the main spike. If these spikes are detected then a pulse radar signal has been detected and the signal can continue to be processed. 
International Journal on Cybernetics \& Informatics (IJCI) Vol. 5, No. 2, April 2016

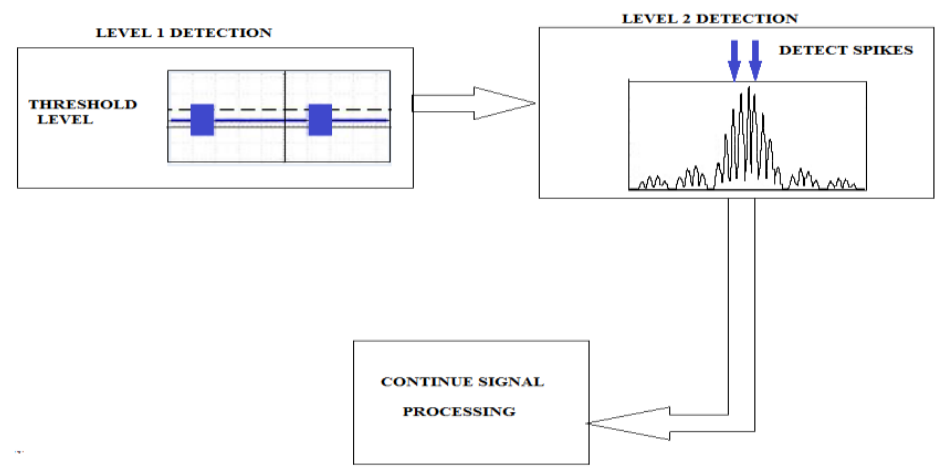

Fig:4 Signal Detection

Level 1 and Level 2 works successively to detect whether a radar signal is been detected. This is to speed up the system.

The illustration of a pulse radar signal is shown in Figure 4. By processing the signal in,time domain characteristics can be determined. The following is the list of outputs and how they relate to Figure

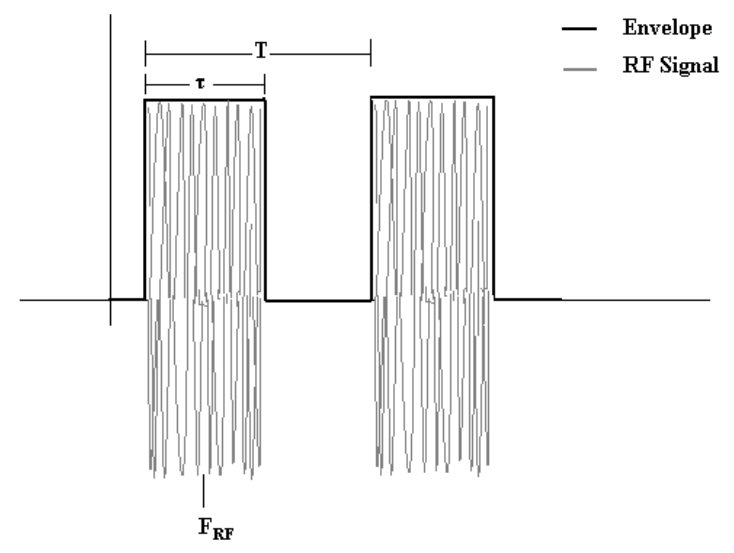

Fig 5 Time representation of the pulsed radar signal

$>\quad \tau$ Gives the Pulse Width

$>\quad$ The inverse of period $\mathrm{T}$ in the time domain gives the Pulse Repetition Rate (PRR)

$>\quad$ The frequency under pulse envelope is the frequency RF denoted as $\mathrm{F}_{\mathrm{RF}}$.

\subsection{EXPERIMENTAL RESULTS}


In Figure 6 the time domain of a sampled signal can be seen. The frequency of the RF signal is $100 \mathrm{MHz}$

$>\quad$ The Local Oscillator frequency is obtained as $99.9 \mathrm{MHz}$

$>\quad$ Calculated Pulse repetition rate is $500 \mathrm{~Hz}$

$>\quad$ Calculated value of Pulse width is $400 \mathrm{uS}$ corresponds to frequency of $2500 \mathrm{~Hz}$.

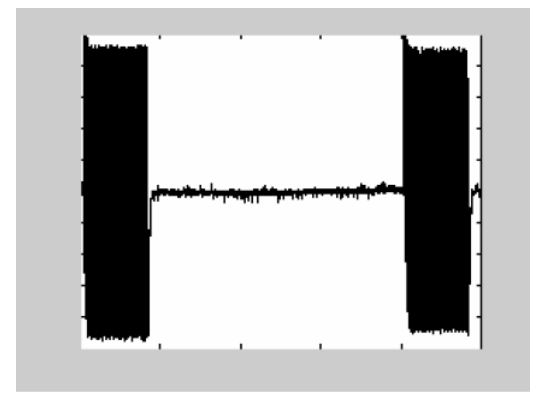

Fig 6 Sampled pulse Radar signal in Mat lab

Figure 7 is the results for the signal in the frequency domain from the FFT, which is used to determine the characteristics of the radar signal.

- $\quad$ The IF frequency is obtained as $100 \mathrm{KHz}$ which is expected.

- $\quad$ Thus, $\mathrm{LO}+\mathrm{IF}=100 \mathrm{MHz}$ which as expected.

- $\quad$ Also the PRR and pulse width are the expected values.

\section{CONCLUSION}

The Project was an overall success. The main objective was to make sure the system was able to automatically scan the entire frequency range and detect and characterize a pulse radar signal within the range. Also there was no error when determining the PRR of the signal. The system could not detect much large pulse width but it doesn't cause much problem because larger pulse width may cause wastage of power. 


\section{ACKNOWLEGMENT}

The author would like to thank Mrs.Renu Agarwal(Scientist Engineer SD), Vikram Sarabhai Space Centre and Anu Mohandas(Assistant professor), Pankaja kasthuri College Of Engineering And Technology for their guidance and support.

\section{REFERENCES}

[1] Couch, Leon W., Digital and Analog Communication Systems, 3rd edition (New Jersey: Prentice Hall, Inc. 1990)

[2] David.M.Pozar, Microwave Engineering New York John Wiley \& sons, second ed.. 1998

[3] David.M.Pozar, Microwave Engineering New York John Wiley \& sons, second ed.. 1998

[4] Haykin, Simon, Communication Systems, 3rd edition (1994)

[5] Jon Hagen, Radio frequency Electronics

[6] Mahafza, Bassem R., Radar Systems Analysis and Design Using Matlab, 2nd edition (New York: Chapman and Hall/CRC 2005)

[7] P.E. Chadwick, High Performance IC Mixers, IEEE Conference on Radio Receivers and Associated Systems, Leeds, 1981, IERE Conference Publication No. 50.

\section{Authors}

Ahalya R S: Received her B.Tech degree in Electronics And Communication engineering from University Of Kerala in 2014. Currently pursuing her M.tech degree in VLSI And Embedded System from Dr.APJ Abdul Kalam Technological University, Kerala.

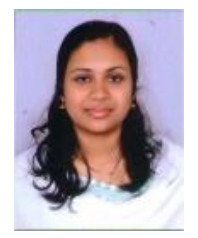

Mary Joseph: received M.Tech Degree in Microwave and Radar from Cochin University of Science and Technology (CUSAT), Kochi, India, in 1997. Currently she is working as Associate Professor in M. A. College of Engineering, Kothamangalam. She has joined in M. A. College of Engineering in 1991 as Assistant Professor. In between she worked at

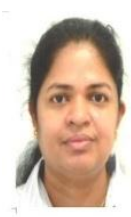
Birla Institute of Science \& Technology-Pilani's (BITS-PILANI) Dubai Campus for 9 years as Assistant Professor during 2000-2008. Her Research interests include Microstrip Antennas and Uniplanar Antennas. 